Dedicated to Scientists

involved in LENR research

\title{
THEORY OF COLD FUSION REACTIONS
}

\section{Gennady V. Mishinsky}

International Intergovernmental Organization Joint Institute for Nuclear Research, http://www.jinr.ru/ 6, Joliot-Curie St., Dubna 141980, Moscow Region, Russian Federation mysh@jinr.ru

Abstract. The reaction of radiative capture of neutron by the nucleus and the nonradiative reaction of cold fusion are based on the same resonant interference exchange interaction. The exchange interaction is manifested not only between identical particles, but also between a particle and its image - an object that has a resonant state with that particle (R-state). The wave function of a particle excites the $\mathrm{R}$-state along its length. The appeared wave function of $\mathrm{R}$-state overlaps and interferes with wave functions of a particle. Thereby, an exchange interaction is generated, which attracts a particle and an object with an R-state to each other. The energy of exchange interaction is an additional contribution to the total energy of overall system simultaneously for all fundamental interactions, including the strong interaction. The exchange energy caused by strong interaction increases the radiative capture cross-section of the neutron by the nucleus, forming a potential pit for it far from the nucleus, and creates a potential well on the slope of the Coulomb repulsion potential for protons or deuterons in case of their resonant interaction with the nucleus. Two protons or two deuterons trapped in such a potential well trigger nonradiative cold fusion.

Keywords: quantum physics, atomic physics, nuclear physics, neutron physics, radiative neutron capture, solid state physics, exchange interaction, resonant interaction, cold nuclear fusion, lowenergy nuclear reactions

PACS: 03.65.-w; 25.60.Pj; 25.40.Ny; 28.20.-v

Bibliography - 49 references

Received 15.05.2019, accepted 20.05.2019

RENSIT, 2019, 11(2):125-142

DOI: $10.17725 /$ rensit.2019.11.125

CONTENTS

1. INTRODUCTION (125)

2. First CF experiments (126)

3. Paricipants of CF Reactions (127)

4. On potential Well and Coulomb barrier (128)

5. INTERACTION OF THERMAL NEUTRONS WITH NUCLEI (130)

6. RESONANT INTERFERENCE EXCHANGE INTERACTION OF NEUTRONS WITH NUCLEI (131)

7. RIEX-INTERACTION OF PROTONS AND DEUTERONS WITH NUCLEI (135)

8. Conclusion (139)

REFERENCES (140)

\section{INTRODUCTION}

Low-energy nuclear reactions [1-6] occurring in a weakly excited condensed matter are divided into two types: cold fusion (CF) reactions and low-energy transmutation reactions of chemical elements (LET or transmutation).

The reactions of $\mathrm{CF}$ include reactions involving hydrogen or deuterium, namely: protons or deuterons, and the nuclei of the main element, for example: palladium, zirconium, nickel, titanium, boron, lithium... These reactions can proceed spontaneously, without external influence. In solids, they occur preferably in samples that have a size of an order of several nanometers. Such a size of samples allows to create a concentration of 2-3 atoms of hydrogen or deuterium per one atom of the main element. The CF reactions can also proceed in solutions and in melts.

Transmutation reactions are realized for all chemical elements, starting with hydrogen, and occur, as a rule, with the participation of a large number of atomic nuclei. The LET reactions include both multinuclear fusion and 
multinuclear fission. They occur only as a result of external influence. Transmutation reactions predominantly occur in melts, in solutions and in dense gases, i.e. on free atoms.

Some experimental similarity in the results of these types of reactions, in the case of $\mathrm{CF}$ reactions, is explained by the initial excitation of the condensed matter due to cold fusion reactions with a subsequent triggering of lowenergy transmutation reactions. The theory of low-energy transmutation reactions proposed by the author of this article is presented in [7-11].

Below we will discuss the mechanism of cold fusion reactions.

\section{FIRST CF EXPERIMENTS}

On March 23, 2019, it turned 30 years since Martin Fleischman and Stanley Pons announced at a press conference that they had executed nuclear fusion at room temperature. The nuclear fusion reaction was implemented by them during the electrolysis of a solution of deuterated lithium hydroxyl in heavy water with a palladium cathode $(0.1 \mathrm{M}$ LiOD in a solution of $\left.99.5 \% \mathrm{D}_{2} \mathrm{O}+0.5 \% \mathrm{H}_{2} \mathrm{O}\right)$ [12]. They reported that a significant amount of excess heat is released during electrolysis, which cannot be explained by chemical reactions. In addition, a weak neutron flux and tritium generation was detected in those experiments. Those results allowed the authors to draw a conclusion about the nuclear origin of excess heat and to assume that the following nuclear reactions take place in palladium cathode:

$$
\begin{aligned}
& \mathrm{d}+\mathrm{d} \rightarrow{ }^{3} \mathrm{He}+\mathrm{n}+3.26 \mathrm{MeV} \\
& \mathrm{d}+\mathrm{d} \rightarrow \mathrm{t}+\mathrm{p}+4.03 \mathrm{MeV} .
\end{aligned}
$$

These reactions are carried out at a low deuteron energy, therefore, thanks to journalists, this phenomenon is called Cold Fusion.

In Russia, the reaction of nuclear synthesis of helium from deuterium was carried out by Filimonenko I.S. [13] thirty years before the press conference of Fleishman M. and Pons S., as early as 1957 . The synthesis took place by the electrolysis of heavy water with a palladium cathode at a temperature of $\sim 1150^{\circ} \mathrm{C}$. Unfortunately, for domestic political reasons, all research on this topic was stopped in 1968. Work was resumed in 1989-1990 after the press conference of Fleischman M. and Pons S. Three therm-emission devices with an electrical power of $12.5 \mathrm{~kW}$ each were recreated. However, the termination of funding for the project led to the loss of both devices and the technology itself. Filimonenko I.S. no longer returned to this work and did not share the technology on this topic.

It should be noted that, perhaps, thanks to the press conference, and not to the "boring" article, the message of Fleischman M. and Pons S. provoked a strong reaction from scientists who began to check their results all over the world. It is noteworthy that from the very beginning of their CF research, physicists and chemists did not limit themselves to electrolysis with a palladiumdeuterium pair, and, due to their facilities and theoretical assumptions about the synthesis mechanism, developed new techniques.

Subsequent experiments on CF performed by other researchers showed that the ratio of the reaction leading to the production of tritium $\mathrm{T}$ (2) to the reaction leading to the production of helium ${ }^{3} \mathrm{He}(1)$ reaches values up to $10^{9}$ [14]. At conventional thermonuclear fusion, both reactions proceed with almost the same probability. McKubre M. wrote: "The production of tritium was not only the first solid evidence for cold fusion - it was the first modern evidence of condensed matter nuclear" [15].

Soon, helium-4 was found in experiments on cold fusion involving deuterium and palladium or other metals, such as titanium, nickel and zirconium [16]. Further studies have shown that the reaction with the release of ${ }^{4} \mathrm{He}$ is the main channel in the process of $\mathrm{CF}$, and the amount of ${ }^{4} \mathrm{He}$ strictly correlates with the amount of excess heat in the reaction:

$\mathrm{d}+\mathrm{d} \rightarrow{ }^{4} \mathrm{He}+23.8 \mathrm{MeV}$ (crystal lattice). 
"Mil's results [16], reported at ICCF-2 in 1991 in Como, Italy, were stunning. When excess heat was present in the electrochemical experiment, helium-4 appeared approximately commensurately in the evolved electrolysis gas. When excess heat was not present, neither was helium-4. Finally, we had a product that made sense thermodynamically and explained the absence of radiation" - stated McKubre M. [15].

In thermonuclear fusion involving two deuterons, the reaction with the release of ${ }^{4} \mathrm{He}$ is known, but a high-energy gamma quantum is always emitted:

$$
\mathrm{d}+\mathrm{d} \rightarrow{ }^{4} \mathrm{He}+\gamma+23.8 \mathrm{MeV} \text {. }
$$

The reaction (4) proceeds at a level of $\sim 10^{-7}$ from the main channels: (1) and (2). No highenergy gamma quanta were detected in the $\mathrm{CF}$ reaction (3).

Note that the replacement of deuterium with hydrogen did not lead, in many experiments, to the appearance of neither excess heat nor the appearance of synthesis products. I.e., no assumed reaction: $\mathrm{p}+\mathrm{p} \rightarrow \mathrm{d}+\mathrm{e}^{+}+\nu_{\mathrm{e}}+0.42$ $\mathrm{MeV}$, other things being equal, was observed.

In [17], "extraneous" chemical elements were produced with a glow discharge in deuterium or hydrogen plasma with a palladium cathode, with predominant synthesis in the case with deuterium. The presence of "extraneous" elements can be explained, as mentioned above, by the initial activation of the cathode surface, due to the reactions of cold fusion followed by the launch of low-energy transmutation reactions.

It should also be recalled that in $1992 \mathrm{~F}$. Piantelli discovered an anomalously high heat release when nickel $\mathrm{Ni}$ was saturated with hydrogen $\mathrm{H}_{2}$ [18, 19]. Subsequently, those experiments formed the basis of technologies for the production of excess heat produced installations of A. Rossi [20].

\section{PARTICIPANTS OF CF REACTIONS}

Obviously, the reaction (3) cannot take place for kinematic reasons. For this reaction to become possible at least one more participant of reaction
"Y" must be available, with which ${ }^{4} \mathrm{He}$ can share energy of $23.8 \mathrm{MeV}$. I.e., reactions (5) or (6) should occur:

$\mathrm{d}+\mathrm{d} \rightarrow{ }^{4} \mathrm{He}+\mathrm{Y}+23.8 \mathrm{MeV}$

$\mathrm{d}+\mathrm{d}+\mathrm{Y} \rightarrow{ }^{4} \mathrm{He}+\mathrm{Y}+23.8 \mathrm{MeV}$.

Let us formulate the requirements for this "Y" participant. First, to get its share of energy, it must be "strongly" coupled to the ${ }^{4} \mathrm{He}$ nucleus. The "Y" participant must create a potential well with a depth of several $\mathrm{MeV}$ for deuterons or with deuterons. Secondly, it must be a heavy, highly ionizing particle, so that when passing through a condensed medium at a short distance from the place where the reaction proceeds, it was able to loose all energy derived from the processes $(5,6)$. The "Y" participant should not fly out of reaction chamber; otherwise it would have been registered by external detectors.

Let us list all possible candidates for the role of "Y" participant: a) atomic electrons; b) crystal lattice - as a whole; c) an unknown particle born during the reaction; d) a neutral, heavy particle with a mass comparable to proton mass constantly present in a medium and which is manifested in CF reactions only; e) a nuclear molecule, which consists of several deuterons $(\geq 3) ; f)$ atomic nucleus.

a) It was shown in [21] that in nuclear fusion reactions occurring in a crystalline solid, the solid-state processes of internal conversion involving both individual electrons and the entire crystal lattice should be taken into account. However, it is impossible to fully explain the course of reaction (3) by the processes of internal conversion due to the inhibition in the redistribution of the released energy due to electromagnetic interaction between ${ }^{4} \mathrm{He}$ and electrons of the crystal lattice only.

b) The energy released as a result of $\mathrm{CF}$ reaction (3) is so great that the effect of receiving at least part of the energy by the entire crystal lattice, similar to the Mössbauer effect, is impossible. 
c) The birth of an unknown, heavy, hadronic particle in a weakly excited condensed medium is not possible for energy reason.

d) The participation of hypothetical neutral or charged heavy hadrons, free or weakly bound to atomic nuclei, at low concentrations in the substance $\left(10^{-14}\right.$ per nucleon) as mediators in $\mathrm{CF}$ reactions [22] cannot explain the high intensity of $\mathrm{CF}$ reactions. In case of their high concentration in the substance, they would have been found long ago in conventional nuclear reactions.

e) To create a nuclear molecule, which consists of several deuterons, a potential well of several $\mathrm{MeV}$ in a solid should be formed. The deuterons themselves are unable to form such a potential well, despite the fact that deuterons are bosons.

Thus, the only candidate remaining from the list of possible CF reactions to the role of " $Y$ " is the atomic nucleus.

It was noted that the intensity of CF reactions and, accordingly, the ${ }^{4} \mathrm{He}$ yield increases with increasing number of deuterium atoms per atom of the main element. This increase is achieved by using metal clusters of a size of several nanometers [23]. The nanoscale metallic cluster has additional electronic energy levels common to all atoms of the cluster. In a solid metal, this is an extended, linear conduction zone. In a cluster, these are separate electronic levels. At absorption of $\mathrm{D}_{2}$ (deuterium)- or $\mathrm{H}_{2}$ (hydrogen)molecules by a cluster, the latter dissociate. And the formed D-, H-atoms lose their electrons, which fill the cluster electronic levels. Thus, in the cluster volume, $\mathrm{d}$ deuterons and $\mathrm{p}$ protons are present in the form of a "gas". They "freely" move in the whole cluster space: they penetrate into the electron shell of main element atom, come close to its nucleus and interact with it and with similar deuterons or protons. The more electrons can be located at cluster levels, the higher is the concentration of protons or deuterons per one atom of main element in the cluster. The concentration of deuterons or protons can reach $300 \%$ (three d or p nuclei per cluster atom) in a wide pressure range with cluster sizes of $\sim 5 \mathrm{~nm}$ (50Á) (Fig. 1) [24]. It is important to prevent clusters from sticking together, which leads to the elimination of additional electronic levels. This can be achieved by adding an insulating composite to the cluster powder of main element, for example, $\mathrm{ZrO}_{2}$ cluster powder.

It is curious that nanoclusters of a 6-7 nm size are formed near metal surfaces, simply as a result of heating the sample to a temperature of $300-400^{\circ} \mathrm{C}$ [25]! Such nanoclusters levitate over a metal surface! If CF reactions occur in the volume of nanoclusters formed by any method, then as a result of the bombardment of metal surface by reaction products, it is locally heated, and both additional local defects and new nanoclusters are produced on it. Emerged "hot" defects and nanoclusters can become centers for the formation of solid structures and the synthesis of "extraneous" chemical elements in low-energy nuclear transmutation reactions $[17$, 20, 26].

\section{ON POTENTIAL WELL AND COULOMB BARRIER}

Since all the above $d+d$ reactions proceed at low energy of deuterons, one of the main problems

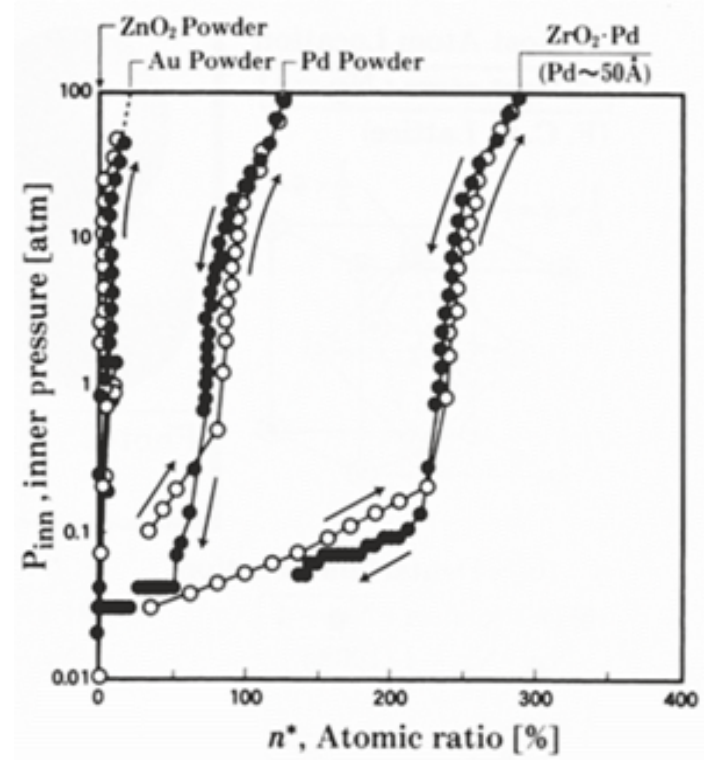

Fig. 1. The concentration of deuterons/protons per atom of the main element $\left(n^{*}\right)$ in a wide range of their pressure for different materials with different grain sizes [24]. 
of cold fusion is the problem of overcoming the Coulomb barrier by deuterons during their fusion.

The reaction (3) is more probable than reaction (2), and reaction (2) is more probable than reaction (1): $\mathrm{W}(3)>\mathrm{W}(2)>\mathrm{W}(1)$. The energies released as a result of these reactions are in the same relation: $23.8 \mathrm{MeV}>4.03 \mathrm{MeV}>$ $3.26 \mathrm{MeV}$. A natural solution to the problem of overcoming the Coulomb barrier and the ratio observed in reaction probabilities would be the fact that a potential well of sufficient depth exists in a condensed matter so that two deuterons or two protons can get into it and stay in it before fusion. Inside such a potential well, protons or deuterons will occupy the same energy state, with minimal potential energy.

Since reactions (1) and (2) proceed through the compound nucleus ${ }^{4} \mathrm{He},\left(\mathrm{d}+\mathrm{d} \rightarrow{ }^{4} \mathrm{He} \rightarrow\right.$ ${ }^{3} \mathrm{He}+\mathrm{n}+3.26 \mathrm{MeV}$ and $\mathrm{d}+\mathrm{d} \rightarrow{ }^{4} \mathrm{He} \rightarrow \mathrm{t}+\mathrm{p}$ $+4.03 \mathrm{MeV}$ ), the $\mathrm{CF}$ process can be considered, in the first approximation, as the process of a certain alpha decay. First of all, deuterons must get into a potential well. The probability of this process is proportional to the probability of one deuteron to get in a potential well and is proportional to the number of deuterons per one atom of main element. Inside the potential well, two deuterons merge to create an $\alpha$-particle $\left({ }^{4} \mathrm{He}\right)$. Further, the formed ${ }^{4} \mathrm{He}$ nucleus tunnels through the potential barrier with decay over channels: (1) $\rightarrow{ }^{3} \mathrm{He}+\mathrm{n}$, (2) $\rightarrow \mathrm{t}+\mathrm{p}$ or $(6) \rightarrow{ }^{4} \mathrm{He}$ (Fig. 2$)$. The probability

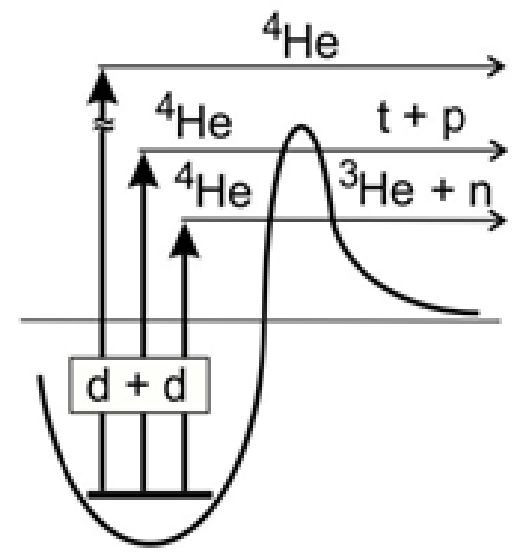

Fig. 2. ${ }^{4} \mathrm{He}$ tunneling through a potential barrier with subsequent decay through channels: ${ }^{4} \mathrm{He} \rightarrow{ }^{3} \mathrm{He}+n,{ }^{4} \mathrm{He}$ $\rightarrow t+p$. of $\alpha$-particle tunneling through the barrier is known to be proportional to the transparency coefficient D:

$$
D=\exp \left[-(2 / \hbar) \int_{r_{1}}^{r_{2}} \sqrt{2 m\left(U_{b}-E\right)}\right],
$$

where $m$ is the mass of $\alpha$-particle, $U_{\mathrm{b}}$ is barrier height, $E$ is the kinetic energy of $\alpha$-particle, which approximately can be considered equal to the decay energy of ${ }^{4} \mathrm{He}$. Since the reaction energy $\mathrm{d}+\mathrm{d} \rightarrow \mathrm{t}+\mathrm{p}+4.03 \mathrm{MeV}$ is greater by $0.77 \mathrm{MeV}$ than reaction energy $\mathrm{d}+\mathrm{d} \rightarrow{ }^{3} \mathrm{He}+$ $\mathrm{n}+3.26 \mathrm{MeV}$, the probability of its realization is also greater. The probabilities ratio of these reactions may be several orders of magnitude. For example, the tellurium isotope Te-106 decays by emitting an $\alpha$-particle with an energy of $E_{\alpha}=4.16 \mathrm{MeV}$ and a half life of $T_{1 / 2}=0.07 \mathrm{~ms}$, and the isotope Te-108 emits an $\alpha$-particle with $\mathrm{E}_{\alpha}=3.32 \mathrm{MeV}$ and with $\mathrm{T}_{1 / 2}$ $=3.15 \mathrm{~s}$ (decay time over $\alpha$-channel). Thus, the probability ratio of $\alpha$-decays for two tellurium isotopes with energy difference $\Delta E=0.84$ $\mathrm{MeV}$ is four and a half orders of $3.15 \mathrm{~s} / 0.07 \mathrm{~ms}$ $=4.5 \cdot 10^{4}$.

Therefore, if the barrier height $U_{b}$ is greater than $3.26 \mathrm{MeV}$, then the reaction $\mathrm{d}+\mathrm{d} \rightarrow \mathrm{t}+\mathrm{p}$ $+4.03 \mathrm{MeV}$ prevails over the reaction $\mathrm{d}+\mathrm{d} \rightarrow$ ${ }^{3} \mathrm{He}+\mathrm{n}+3.26$. And the reaction $\mathrm{d}+\mathrm{d} \rightarrow{ }^{4} \mathrm{He}+$ "Y" + 23.8 MeV becomes dominant, firstly, due to the huge energy release and, secondly, due to the absence of further decay of ${ }^{4} \mathrm{He}$ compound nucleus (Fig. 2).

However, the crystalline lattice of a solid does not create potential well of such depth. Such a well is formed as a result of resonant interference exchange interaction of the atomic nucleus with deuteron or proton.

\section{INTERACTION OF THERMAL NEUTRONS WITH NUCLEI}

Rukhadze A.A. and Grachev V.I. wrote in the article: "LENR in Russia" [27]: "From the first steps of nuclear physics, its development has been largely, in General, in two directions - of highand low-energetic. E. Rutherford (1871-1937), the 
"father" of nuclear physics who discovered (1919) artificial "transmutation of elements" - nuclei ( ${ }_{7}^{14} \mathrm{~N}+{ }_{2}^{4} \mathrm{He} \rightarrow{ }_{8}^{17} \mathrm{O}+{ }_{1}^{1} \mathrm{H}$ ), by fast alpha particles, have interpreted this "modern alchemistry" as the area of high-energy processes, which opening the era of nuclear power. Available by the time the works on nuclear transmutations of stable isotopes in natural conditions, and primarily the receipt by the american chemists $K$. Irion and J. Wendt (1922) [28, 29], of helium in alphadecay of tungsten, induced of electric explosion, Rutherford declared as error. After such a statement by E.Rutherford, the low-energy trend faded away and "cold" nuclear transmutation became "pseudoscience" for many years."

However, the low-energy processes, of course, could not disappear nowhere, they simply "disguised" themselves as high-energy processes. One such world-famous process that laid the foundation for creating an entire industry is the process of resonant absorption of a thermal neutron by atomic nucleus.

Such phenomena as resonant radiation capture of neutron by nucleus and nuclear fission by a thermal neutron are widely known. As a result of the radiation neutron capture, an energy of $\sim 8 \mathrm{MeV}$ is released in the reaction. In this case, several gamma quanta are emitted with an average energy of $\sim 2 \mathrm{MeV}$. In these reactions, radioactive isotopes are often formed, experiencing $\beta^{-}-$или $\beta^{+}$-decay. In the process of fission of heavy nuclei by thermal neutrons, prompt gamma quanta, neutrons are emitted, and two radioactive fragments are formed. The energy released in the process of nuclear fission, for example: ${ }^{235} \mathrm{U}+$ $\mathrm{n}$, is $\sim 200 \mathrm{MeV}$, and its fission cross-section by a thermal neutron is 586 barn.

Both of these processes with thermal neutrons are high-energy processes on the output channel. Perhaps that is why there is no comparison of reactions of cold nuclear fusion with reactions of radiative capture of a thermal neutron and, especially, with nuclear fission in the scientific LENR-literature. However, the ideology associated with the screening of a proton by an electron: the "transformation" of a proton into a neutron is present in theoretical models of the CF [30, 31].

In the input channel, both reactions with thermal neutrons are low-energy reactions. And the fission of a nucleus by a thermal neutron can be called, just right, a "cold" nuclear fission. Indeed, all three processes: radiation neutron capture, and "cold" nuclear fission, and cold fusion are low-energy processes in the input channel, but high-energy processes in the output channel. The only difference is that in the first two cases a thermal neutron participates in the input channel, and either a thermal proton or thermal deuteron participates in the third case.

The process of radiative capture of a slow neutron with the formation of a compound nucleus has a pronounced resonant character when the neutron energy is close to one of the values, corresponding to quasistationary state of the compound system: the neutron plus the target nucleus [32]. The energy level of neutron resonance $\mathrm{R}$-state is characterized by the energy width $\Gamma$, which is inversely proportional to its lifetime $\tau=\hbar / \Gamma$. The level widths of compound nuclei at excitation energies close to the neutron binding energy are usually small compared with the distances between them. The full width of the level $\Gamma$ consists of two parts: the radiation width $\Gamma_{\gamma}$ and the neutron width $\Gamma_{\mathrm{n}}$. The radiation width corresponding to slow neutron capture is $\Gamma_{\gamma} \sim 0.1 \mathrm{eV}$. The neutron width $\Gamma_{\mathrm{n}}$ at low neutron energies is proportional to its speed and can be much smaller than the radiation width. Thus, the full width of nuclear levels $\Gamma=\Gamma_{\gamma}+\Gamma_{\mathrm{n}}$ at excitation energies close to neutron binding energy has the order $\Gamma \sim 0.1 \mathrm{eV}$. The distances between levels, as shown by numerous experimental data, have values of $\sim 10 \mathrm{eV}$.

To characterize radiation capture, the dependence of neutron absorption cross-section on its energy $\sigma(E)$ is usually used. The effective capture cross-section of a slow neutron in the presence of a single resonance level, which corresponds to the resonant energy value $E_{0}$ and of the width $\Gamma_{\gamma}, \Gamma_{\mathrm{n}}$, is described by the Breit-Wigner formula: 


$$
\sigma=g \pi \lambda_{0} \lambda \frac{\Gamma_{n} \Gamma_{\gamma}}{\left(E-E_{0}\right)^{2}+\Gamma^{2}},
$$

where $g=\frac{2 j+1}{2(2 i+1)}, i-$ nuclear spin, $j=i \pm 1 / 2$ - compound nuclear moment, $\lambda_{0}-$ neutron wavelength in accordance with energy $E_{0}, \lambda-$ neutron wavelength $\lambda=\hbar / m_{n} v, m_{n}, v-$ neutron mass and speed. The peak value of capture crosssection at neutron energy $E=E_{0}$ is equal to $\sigma_{0}\left(E_{0}\right)=\lambda_{0}^{2}\left[g \pi \frac{\Gamma_{n} \Gamma_{\gamma}}{\Gamma^{2}}\right]$.

Experiments on resonant capture of thermal neutrons show that at energies slightly higher than the neutron binding energy, the number of $R$-levels is significant, and the distances between them become much less than $1 \mathrm{eV}$. As is known, the majority of isotopes in the region of thermal energy of neutrons show noticeable crosssections of their capture. There are isotopes, which cross-sections are equal to tens to hundreds of thousands of barn. If these crosssections $\sigma_{\mathrm{T}}$ are reduced to potential interaction radii $R_{\mathrm{T}}\left(\sigma_{\mathrm{T}}=4 \pi R_{\mathrm{T}}{ }^{2}\right)$, then it turns out that the radii $R_{T}$ are also thousands or hundreds of thousands times greater than the isotope nuclei radii $R_{\text {nucl }}$ (see Table). Such increased values of cross-sections are connected with a large number of closely spaced, resonant $R$-levels and are explained by resonant interference exchange interaction (RIEX-interaction).

\section{RIEX-INTERACTION OF NEUTRONS WITH NUCLEI}

This article argues that exchange interaction takes place both between identical particles and between a particle and a resonant $R$-state.

Thermal neutron capture parameters, $\lambda \approx 2 \cdot 10^{5} \mathrm{fm}$.

\begin{tabular}{|c|c|c|c|}
\hline Isotope & $\sigma_{\mathrm{T}^{\prime}}$ barn & $\mathrm{R}_{\text {nucl }}, \mathrm{fm}$ & $\mathrm{R}_{\mathrm{T}}, \mathrm{fm}$ \\
\hline${ }^{6} \mathrm{Li}$ & 940 & 2.4 & $1.2 \cdot 10^{4}$ \\
\hline${ }^{10} \mathrm{~B}$ & 3840 & 2.8 & $2.5 \cdot 10^{4}$ \\
\hline${ }^{64} \mathrm{Ni}$ & 1.49 & 5.2 & 340 \\
\hline${ }^{113} \mathrm{Cd}$ & $2.06 \cdot 10^{4}$ & 6.3 & $4.1 \cdot 10^{4}$ \\
\hline${ }^{135} \mathrm{Xe}$ & $2.65 \cdot 10^{6}$ & 6.7 & $4.6 \cdot 10^{5}$ \\
\hline${ }^{157} \mathrm{Gd}$ & $2.54 \cdot 10^{5}$ & 7.0 & $1.4 \cdot 10^{5}$ \\
\hline${ }^{235} \mathrm{U}$ & 586 & 9.3 & $6.8 \cdot 10^{3}$ \\
\hline \multicolumn{4}{|r}{} \\
\hline
\end{tabular}

Neutrons trapped in any medium and interacting with its nuclei are finally thermalized, acquire the temperature of that medium. At room temperature $T=300 \mathrm{~K}$, the average neutron energy is $E=0.025 \mathrm{eV}$, and the neutron de Broglie wavelength is $\lambda_{\mathrm{n}}=\mathrm{h} / \mathrm{mv} \approx 2 \cdot 10^{5} \mathrm{fm}$. Since neutrons have no charge, they move freely in the medium, representing a neutron "gas". If a compound nucleus has a resonance state with a neutron, then it begins to interact resonantly with that $R$-state.

The resonant $R$-state present in the nucleus is a certain analog of the neutron, its "image". This "image" is "identical" to neutron. The "image" becomes real when the neutron excites the R-state at its wavelength (Fig. 3). Thus, the neutron is simultaneously in two states: in its own state $\psi_{n}(n)$ and partially in the R-state $\psi_{R}(n)$. Accordingly, the $R$-state is also in two states: in its own state $\psi_{R}(R)$ and partially in the neutron state $\psi_{n}(R)$. The appeared $R$-state wave function overlaps and interferes with the neutron wave function. Thus, a resonant interference exchange interaction is generated.

As is well known, there is an exchange interaction in quantum physics between identical particles, which is characterized by exchange energy [33]. The more wave functions of identical particles overlap, the greater is the exchange energy value. The exchange interaction between

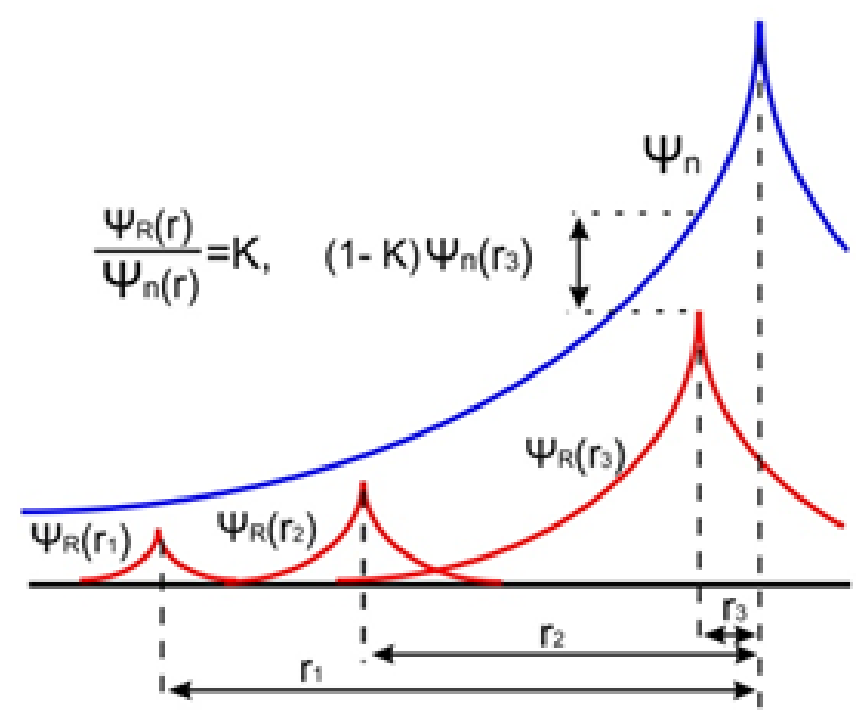

Fig. 3. The wave function of the R-state depending on the distance to the neutron. 
identical particles can have a different character: to have either attraction potential or repulsion potential. Since the exchange interaction energy is an additional contribution to the total energy of overall system, this contribution can be both negative and positive. So, this contribution is positive in a parahelium atom, in which electron spins are antiparallel, - electrons repel each other, and this contribution is negative in an orthohelium atom with parallel spins of electrons - electrons attract each other. When two hydrogen atoms interact, on the contrary, hydrogen atoms with parallel electron spins repel each other, and they attract each other with antiparallel electron spins. Such a difference is explained by the fact that, in the helium atom, the exchange integral of two electrons has a positive value, and for two hydrogen atoms the exchange integral of their electrons has a negative value. The latter is associated with the Coulomb interaction of electrons not only among themselves, but also with the protons of neighboring hydrogen atoms [34].

The exchange energy of electrons is caused by their electromagnetic interaction. But the neutron is a neutral particle. However, the exchange energy is an additional contribution to the total energy of the system at the same time for all fundamental interactions, and not only for the electromagnetic interaction. The first correction to the system energy $\Delta E^{(1)}$ is calculated in the perturbation theory by means of equation (8). All fundamental interactions always and simultaneously stand in the perturbing potential of interacting particles, including identical particles or other "identical objects": strong interaction $F$, electromagnetic interaction EM, weak interaction $W$ and gravitational interaction G.

$$
\Delta E^{(1)}=\frac{\int \psi^{*}(A, B)[F, E M, W, G] \psi(A, B) d V_{A} d V_{B}}{\int \psi^{*}(A, B) \psi(A, B) d V_{A} d V_{B}}
$$

where $\psi(A, B)=\left[\psi_{A}^{V} \psi_{B}^{V}\right]\left\{S_{A} S_{B}\right\}$ is full wave function of two resonant interacting, "identical objects" $A$ and $B$, which consists of spacial $\left[\psi_{A}^{V} \psi_{B}^{V}\right]$ and spin $\left\{\mathrm{S}_{\mathrm{A}} \mathrm{S}_{\mathrm{B}}\right\}$ parts. $d V_{A}=d x_{A} d y_{A} d \tau_{A}, d V_{B}=d x_{B} d y_{B} d \tau_{B}$ is elementary spatial volume for objects $A$ and $B$. The denominator has normalizing integral.

The spatial region of exchange interaction for all fundamental potentials is determined by the lengths of wave functions of any resonantly interacting "identical objects" $A$ and $B$, since then they are simultaneously in two states: proper state $\psi_{a}(\mathrm{~A}), \psi_{b}(\mathrm{~B})$ and identical state $\psi_{b}(\mathrm{~A}), \psi_{a}(\mathrm{~B})$. The proper part $\psi_{a}(\mathrm{~A})$ interacts in its place $a$ with the identical part $\psi_{a}(\mathrm{~B})$, and the proper part $\psi_{b}(\mathrm{~B})$ interacts in its place $b$ with $\psi_{b}(\mathrm{~A})$. Thus, due to the resonant interference exchange interaction, the short-range strong and local weak interactions become "long-range" interactions.

For interacting neutron and $R$-state, their full wave function $\psi(n, R)=\left[\psi_{n}^{V} \psi_{R}^{V}\right]\left\{S_{n} S_{R}\right\}$ and the potential making the main contribution to the exchange energy is the strong interaction $F$ :

$$
\Delta E_{n R}^{(1)}=\frac{\int \psi^{*}(n, R)[F] \psi(n, R) d V_{n} d V_{R}}{\int \psi^{*}(n, R) \psi(n, R) d V_{n} d V_{R}} .
$$

Since the total wave function of two fermions should be an antisymmetric function, either the spatial wave function $\left[\psi_{n}^{V} \psi_{R}^{V}\right]$ should be antisymmetric and the spin wave function $\left\{\mathrm{S}_{\mathrm{n}} \mathrm{S}_{\mathrm{R}}\right\}$ symmetric $(10 a, b, c)$, or vice versa $(10 d)$. Thus, for neutron and $R$-state, the following combinations are acceptable:

$$
\begin{aligned}
& {\left[\psi_{n}(n) \psi_{R}(R)-\psi_{n}(R) \psi_{R}(n)\right]\left[\left\{S^{+}(n) S^{+}(R)\right\},\right.} \\
& {\left[\psi_{n}(n) \psi_{R}(R)-\psi_{n}(R) \psi_{R}(n)\right]\left[\left\{S^{-}(n) S^{-}(R)\right\},\right.} \\
& {\left[\psi_{n}(n) \psi_{R}(R)-\psi_{n}(R) \psi_{R}(n)\right] \times} \\
& \times\left\{S^{+}(n) S^{-}(R)+S^{-}(n) S^{+}(R)\right\} \\
& {\left[\psi_{n}(n) \psi_{R}(R)+\psi_{n}(R) \psi_{R}(n)\right] \times} \\
& \times\left\{S^{+}(n) S^{-}(R)-S^{-}(n) S^{+}(R)\right\}
\end{aligned}
$$

The energy of a strong perturbation $F$ depends on spin variables. However, suppose that the perturbation $F$ does not change the orientation of neutron spin and $R$-state. Therefore, when averaging the spin wave functions in the numerator and the denominator (9), the same factors appear, which are reduced. 
As a result, $\psi(n, R)$ corresponds to the parts of wave functions (10), depending on coordinates $\psi(n, R)=\left[\psi_{n}^{V} \psi_{R}^{V}\right]$ only. Then:

$$
\begin{aligned}
& {\left[\psi_{n}^{V} \psi_{R}^{V}\right]^{*}\left[\psi_{n}^{V} \psi_{R}^{V}\right]=\psi_{n}^{*}(n) \psi_{R}^{*}(R) \psi_{n}(n) \psi_{R}(R)+} \\
& +\psi_{n}^{*}(R) \psi_{R}^{*}(n) \psi_{n}(R) \psi_{R}(n) \pm \\
& \pm\left[\psi_{n}^{*}(n) \psi_{R}^{*}(R) \psi_{n}(R) \psi_{R}(n)+\right. \\
& \left.+\psi_{n}^{*}(R) \psi_{R}^{*}(n) \psi_{n}(n) \psi_{R}(R)\right] .
\end{aligned}
$$

The function integral in square brackets is the exchange integral. The plus sign in front of the square bracket in equation (11) corresponds to the symmetric coordinate wave function $(10 d)$, the minus sign corresponds to antisymmetric wave function in $(10 a, b, c)$.

The integrals of the first two terms (11) characterize the strong interaction $F$ at a distance exceeding its action between neutron and $R$-state, as well as between the part of neutron in $R$-state and part of $R$-state in the neutron. Therefore, these integrals are equal to zero.

$$
\begin{aligned}
& \int\left|\psi_{n}(n)\right|^{2} F\left|\psi_{R}(R)\right|^{2} d V_{n} d V_{R}=0, \\
& \int\left|\psi_{n}(R)\right|^{2} F\left|\psi_{R}(n)\right|^{2} d V_{n} d V_{R}=0 .
\end{aligned}
$$

Thus, we can consider that in the numerator (9) for strong interaction:

$$
\begin{aligned}
& {\left[\psi_{n}^{V} \psi_{R}^{V}\right]^{*}\left[\psi_{n}^{V} \psi_{R}^{V}\right]=} \\
& = \pm\left[\psi_{n}^{*}(n) \psi_{R}^{*}(R) \psi_{n}(R) \psi_{R}(n)+\right. \\
& \left.+\psi_{n}^{*}(R) \psi_{R}^{*}(n) \psi_{n}(n) \psi_{R}(R)\right] .
\end{aligned}
$$

Potential $F$ is negative. The exchange integral in this case also has a negative value. Therefore, a neutron ( $\operatorname{spin} s=1 / 2$ ) and a nucleus with $\mathrm{R}$-state are attracted to each other when their spatial wave function is symmetric (plus in front of square bracket in (12)) and their spins are antiparallel.

The intensity of neutron excited R-state, and, consequently, the amplitude of its wave function $\psi_{R}(r)$ is determined by the amplitude of the neutron wave function $\psi_{n}(r)$ in the region of nucleus at a distance $r$ from it (Fig. 3) and is proportional to some coefficient $\mathrm{K}$ : $\psi_{R}(r)=\mathrm{K} \psi_{n}(r)$ or $\psi_{R}(R)=K \psi_{n}(n)$. As the neutron approaches the nucleus, the intensity of the R-state will increase. The overlap of the neutron wave functions and the R-state $\psi_{n}(r) \psi_{R}(r)=K\left|\psi_{n}(r)\right|^{2}$. will increase. It can be seen that the overlap increases in proportion to the neutron density $\left|\psi_{n}(r)\right|^{2}$ at a distance $r$ from nucleus.

The value of coefficient $K$ depends on the affinity of $R$-state with neutron. This affinity is determined, in addition to the coincidence of their energy, by coincidence of their spins, parities. The value of coefficient $K$ also depends on the oscillator strength of $R$-state. Thus, the coefficient $K$ depends on the properties of a particular isotope with which the neutron interacts. For two neutrons and two protons in identical states, the coefficient $K$ equals to one $K=1$. Due to the charge independence of the strong interaction, $K=1$ for neutron-proton pair and for deuteron. For interacting neutron and $R$-state, the coefficient $K$ for most isotopes will generally be less than one $K<1$. It can be greater than one $K>1$, in case when the $R$-state has a collective nature.

The wave function of R-state $\psi_{R}(r) \equiv \psi_{R}(R)$ will be present in the neutron state $\psi_{n}(R)$ with the same coefficient $K$ : $\psi_{n}(R)=K \psi_{R}(R)=K^{2} \psi_{n}(n), \quad \psi_{R}(R) \equiv \psi_{R}(n)$. The overlap of their wave functions, $\psi_{n}(R) \psi_{R}(R) \equiv \psi_{n}(R) \psi_{R}(n)=K^{3}\left|\psi_{n}(n)\right|^{2} \equiv K^{3}\left|\psi_{n}(r)\right|^{2}$, and it also increases in proportion to the neutron density $\left|\psi_{n}(r)\right|^{2}$ at a distance $r$ from the nucleus. Consequently, the integral of (12) and the energy of the exchange interaction $\Delta E^{(1)}(r)(9)$ are written: $\Delta E_{n R}^{(1)} \sim 2 \int K\left|\psi_{n}(r)\right|^{2} F\left|\psi_{n}(r)\right|^{2} K^{3} d^{2} V_{n}$

As strong interaction is a short-range interaction, the integral (13) is important only in a volume of nucleus $V_{A}$, whose diameter is equal to ØA. Therefore, the action magnitude of $F$ in the exchange interaction of neutron with $R$-state on the entire interval $L$ - from the start of this interaction to the nucleus, will be considered constant: $F_{R}=$ const. I.e., $F_{R}$ does not depend on the distance $r$ between the neutron and the nucleus. The gap $L$ is related to neutron wavelength as $L \sim K^{4} \cdot \lambda_{\mathrm{n}}$. 
Since $\psi_{n}(r)>>\varnothing \mathrm{A}$, than $\psi_{n}(r)=$ const in a volume equal $V_{A}$. It follows from (13) that exchange interaction energy $\Delta E^{(1)}(r)$ is proportional to squared neutron density in the region of nucleus $\left|\psi_{n}(r)\right|_{V_{A}}^{2}$ when the neutron is located at a distance $r$ to it.

$$
\begin{aligned}
& \Delta E_{n R}^{(A)}(r) \sim 2 K^{4} \int_{V_{A}}\left|\psi_{n}(r)\right|^{2} F_{R}\left|\psi_{n}(r)\right|^{2} d^{2} V_{n}, \\
& \Delta E_{n R}^{(A)}(r) \sim 2 F_{R} K^{4}\left|\psi_{n}(r)\right|^{4} \int_{V_{A}} d^{2} V_{n} .
\end{aligned}
$$

The integral in (14) is squared nuclear volume. It is proportional to squared mass number of the nucleus $\sim \rho M^{2}$. Let's introduce the notation $U$, which includes $2 F_{\mathrm{R}}, \varrho$ and the normalization integral (9).

$$
\Delta E_{n R}^{(A)}(r)=U K^{4} M^{2}\left|\psi_{n}(r)\right|^{4} .
$$

Let's estimate the value of $U$, which characterizes the potential of strong interaction. In the theory of a deuteron, at nuclear potential well radius of $1.45-1.7 \mathrm{fm}$, the well depth is 50$35 \mathrm{MeV}$. This value characterizes the potential of a neutron-proton strong interaction. In the deuteron, the neutron density is $\left|\psi_{n}(r)\right|^{2}=1$, the coefficient $K=1$, and $M^{2}=4$. Then $U=$ 9-12 MeV.

When a neutron interacts with $R$-state, its wave function is transformed from a plane wave into a bound state wave function. The orbital moment of a thermal neutron interacting with nucleus is $\ell=0$. Therefore, we represent the neutron wave function $\psi_{n}(r)$ in the form of radial part of the $S$-wave function of electron located on orbital with radius $a_{0}$, which rotates around a nucleus with quantum numbers $n=1$ and $\ell=0$. The density distribution of such an electron in the radial direction is characterized by function $P(r)=C\left[r^{2} \exp \left(-2 r / a_{0}\right)\right]$, where $C$ is normalization constant, $a_{0}$ is electron orbital radius [34]. The red line in Fig. 4 represents, in relative units, the values of the function from nucleus to $a_{0}$ (500 $\mathrm{fm})$; the green line gives the neutron density in the region of nucleus when neutron is at a distance $r$ from nucleus: $\left|\psi_{n}(r)\right|^{2}=P\left(a_{0}-r\right)$ and the blue line gives the exchange interaction potential

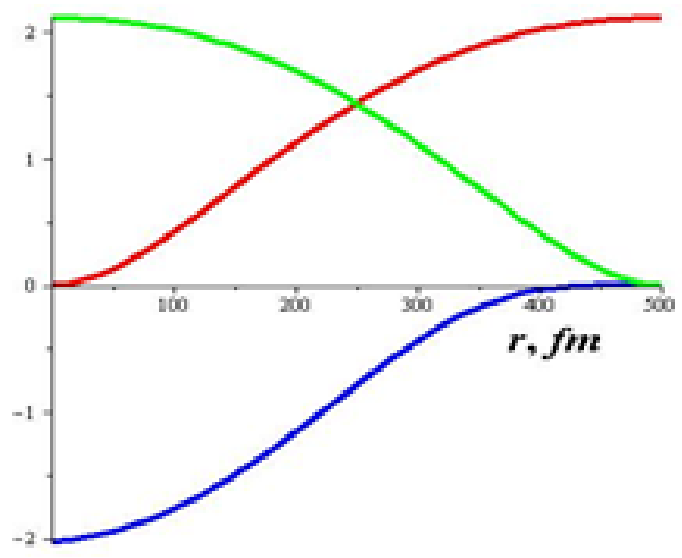

Fig. 4. The red line is the radial density of the orbital electron; green - the density of a neutron located at a distance r from the nucleus; blue - RIEX potential.

$\Delta E_{n R}^{(1)}(r)=-\left[P\left(a_{0}-r\right)\right]^{2}$ (the values $a_{0}=500 \mathrm{fm}$ and $U K^{4} M^{2}=-2 \mathrm{MeV}$ are chosen arbitrarily).

Thus, when a neutron interacts with $\mathrm{R}$-state at a distance of $L \sim K^{4} \cdot \lambda_{\mathrm{n}}$ to the nucleus, a potential pit is formed, which depth is equal to $U K^{4} M^{2}$ (Fig. 5). Distance $L$ can also be correlated with potential interaction radius $R_{\mathrm{T}}$ (see Table). The red line in Fig. 5 represents the nuclear potential of nickel, for example, ${ }^{64} \mathrm{Ni}$ c $\mathrm{R}_{\mathrm{T}}=340 \mathrm{fm}$ (see Table), the blue line represents the potential of RIEX interaction, and the green line represents the summarized potential (the value $U K^{4} M^{2}=$ $-2 \mathrm{MeV}$ is chosen arbitrarily).

Once in the potential pit, the neutron is in a region below neutron binding energy, where the density of neutron energy levels is significant, and, therefore, the resonant interaction of neutron with them is also large. Thus, the neutron "rolls", like downhill, along a potential pit into a potential well of the nucleus. Consequently,

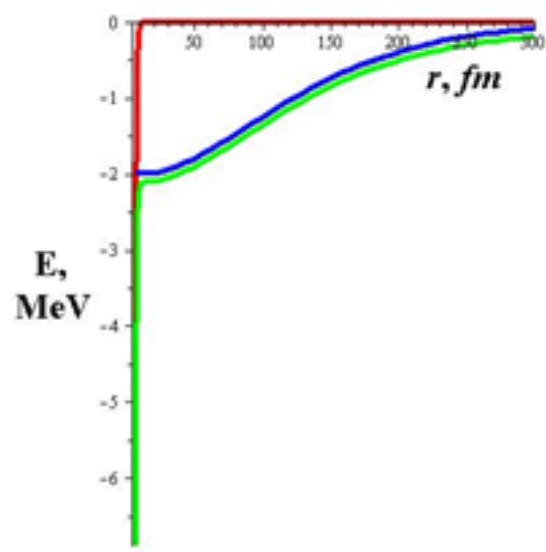

Fig. 5. Red line - nuclear potential Ni; blue - RIEX potential Ni-n; green - total potential Ni+n. 
the neutron is captured by nucleus, due to the resonant interference exchange interaction, at a distance $L \approx R_{\mathrm{T}}$.

\section{RIEX INTERACTION OF PROTONS AND DEUTERONS WITH NUCLEI}

Unlike neutron, proton and deuteron have electric charge that prevents them from approaching the nucleus. On the way to the nucleus, proton and deuteron meet a Coulomb barrier. If for resonant neutron capture it is necessary for the corresponding compound nucleus to have resonant $R$-states, then for the proton and, apparently, for the deuteron behind the Coulomb barrier of the compound nucleus there is a large number of resonance levels, up to the height of Coulomb barrier (Fig. 6). A proton, once in resonance with one of these R-levels, begins to RIEX-interact with it. It is noteworthy that the proton energy can be from thermal energy to energy equal to the height of Coulomb barrier. Therefore, losing energy when approaching the nucleus, the proton, thereby, scans the energy levels of nucleus beyond the Coulomb barrier, until it gets into resonance with a suitable R-level (Fig. 6). The resultant, due to the exchange interaction, attraction force of the proton to the nucleus, increases the proton energy, bringing it out of resonance. The appeared Coulomb braking returns the proton back into resonance with R-level. Thus, the

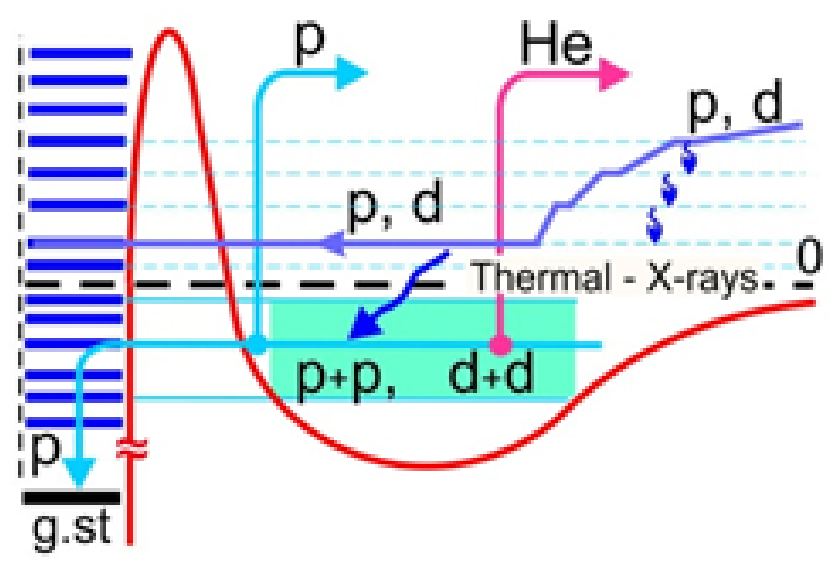

Fig. 6. The levels of the compound nucleus $N+p$ (d) beyond the Coulomb barrier and the interaction with them of a proton or deuteron. energy of a proton that got into resonance with a suitable $R$-level remains constant.

For interacting proton and $R$-state, their total wave function is $\psi(p, R)=\left[\psi_{p}^{V} \psi_{R}^{V}\right]\left\{\psi_{p}^{S} \psi_{R}^{S}\right\}$. Just like a neutron, a proton ( $\operatorname{spin} s=1 / 2$ ) and a nucleus with $R$-state are attracted to each other when their spins are antiparallel and their spatial wave function is symmetric

And since proton and deuteron have an electric charge equal to one +1 , then both strong $F$ and electromagnetic, namely Coulomb EM = $k \cdot q^{2} / r_{\text {pN }}$, interactions will make up the disturbing potential:

$\Delta E_{p R}^{(1)}=\frac{\int \psi^{*}(p, R)[F, E M] \psi(p, R) d V_{p} d V_{R}}{\int \psi^{*}(p, R) \psi(p, R) d V_{p} d V_{R}}$.

Since strong interaction does not depend on the charge, than

$$
\begin{aligned}
& \Delta E_{p R}^{(1)}=\Delta E_{F}^{(1)}+\Delta E_{E M}^{(1)}=\frac{\int \psi^{*}(p, R)[F] \psi(p, R) d V_{p} d V_{R}}{\int \psi^{*}(p, R) \psi(p, R) d V_{p} d V_{R}}+ \\
& +\frac{\int \psi^{*}(p, R)\left[k q^{2} / r_{p N}\right] \psi(p, R) d V_{p} d V_{R}}{\int \psi^{*}(p, R) \psi(p, R) d V_{p} d V_{R}} \approx \Delta E_{F}^{(1)},
\end{aligned}
$$

where $k=1 / 4 \pi \varepsilon_{0}=8.99 \cdot 10^{9} \mathrm{~N} \cdot \mathrm{m}^{2} / C^{2}\left(\varepsilon_{0}=\right.$ $8.85 \cdot 10^{-12} \mathrm{~F} / m$-electric constant $), r_{\mathrm{pN}}$ is a distance between proton and nucleus, $q=1.6022 \cdot 10^{-19}$ $C$ is proton charge. Note that the potentials of strong and electromagnetic interaction have opposite signs.

Let us estimate the energy contribution of Coulomb interaction to the exchange energy. The ground state of parahelium atom $2 E+C$ $+A, 1^{1} S_{0}$ has energy of $79.0 \mathrm{eV}$. " $A$ " is exchange energy. " $C$ " is the energy of usual Coulomb repulsion of electrons. $2 \mathrm{E}_{\mathrm{a}}$ is the electron energy without taking into account their interaction, $2 \mathrm{E}_{\mathrm{a}}$ $=2 \cdot 54.4=108.8 \mathrm{eV}$ is the doubled ionization energy of helium ion $\mathrm{He}^{+}$. Electrons in $1^{1} S_{0}$ are in the same state and for them " $A$ " $=$ " $C$ " = $15 \mathrm{eV}$ [34]. The parahelium diameter is $D_{\mathrm{He}}=$ $62000 \mathrm{fm}$. As will be seen below, thanks to the RIEX interaction, a proton (deuteron) creates a bound, nuclear molecule with the target nucleus (Fig. 6). Estimates showed that the radius of the 
proton orbitals in a nuclear molecule (Fig. 7) is, for different nuclear charges, in the range $R_{p}$ $=50-150 \mathrm{fm}$ for $U K^{4} M^{2} \sim-2 \mathrm{MeV}$. It is clear that bottom position and potential well depth depend on the value $U K^{4} M^{2}$. The ratio $D_{\mathrm{He}} / R_{\mathrm{p}}$ is three orders of magnitude; accordingly, the ratio in energies will have the same value. Thus, the energy contribution of the exchange Coulomb interaction $\Delta E_{E M}^{(1)} \sim 15 \mathrm{keV}$ to the total exchange energy, as compared with the strong interaction $\mathrm{UK}^{4} M^{2} \sim-2 \mathrm{MeV}$, can be neglected. Energy contributions from weak $W$ and gravitational $G$ interactions can also be neglected. But it should be remembered that they are always present in the perturbing potential of exchange interaction.

Let us produce for the proton or deuteron with wave function $\psi_{p}(r)$ the same procedures as for the neutron, relative to the energy of its exchange interaction with the $R$-state. Then $\Delta E_{p R}^{(A)}=U K^{4} M^{2}\left|\psi_{p}(r)\right|^{4}$. As a result, a "exchange" potential well forms at the Coulomb barrier (Fig. 7). It should be emphasized that the emerging nuclear molecule is formed as a result of a strong interaction between proton and nucleus, and not due to the electromagnetic, collective response of internal atomic electrons, which shield the slow proton charge [35]. The red line in Fig. 7 represents the nuclear potential of nickel $\mathrm{Ni}$ plus Coulomb potential $\mathrm{Ni}+\mathrm{p}$, the blue line represents the RIEX potential (values

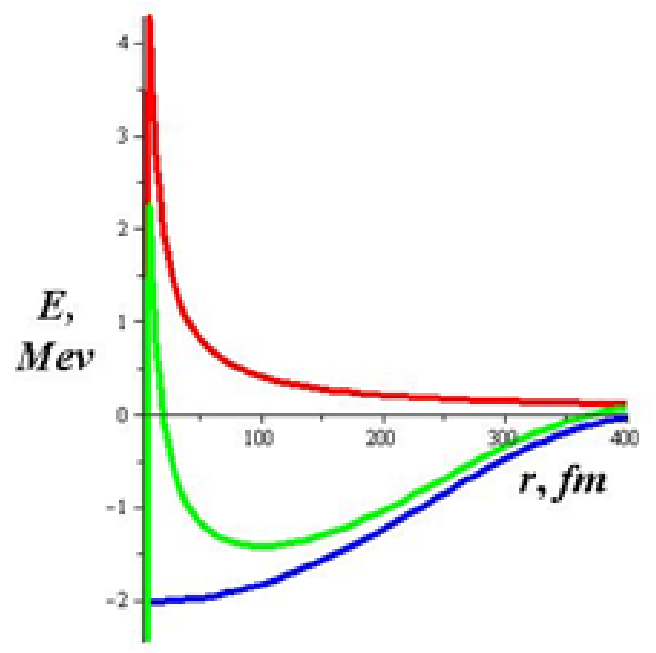

Fig. 7. Red line - nuclear plus Coulomb potentials Ni+p; blue - RIEX potential Ni-p; green - total potential Ni+p.
$400 \mathrm{fm}$ and $U K^{4} M^{2}=-2 \mathrm{MeV}$ are chosen arbitrarily) and the green line shows summarized potential.

Such a picture resembles the double-humped barrier present in spontaneous fission isomers (SFI) in the region of nuclei of transuranium chemical elements with a nuclear charge of 92 to 97 [36]. The states of spontaneous fission isomers are characterized by a high degree of deformation. Therefore, sometimes SFI are called shape isomers. The nature of SFI is associated with nuclear shell corrections, which give their energy contribution, in calculating the potential fission barrier for heavy nuclei, which are performed in the framework of the droplet model. The excitation energy of shape isomers is from 2 to $4 \mathrm{MeV}$, and the half-lives vary from nanoseconds to milliseconds.

Unlike SFI, which potential well is above the potential energy zero line, the exchange potential well is located below the zero line. Therefore, a proton or deuteron that hits it, remains in it and creates a nuclear molecule with a target nucleus. Another important condition is needed for the formation of nuclear molecule: it is necessary that the nucleus on the inside of its Coulomb barrier has resonant $R$-states with a proton (deuteron) located in the well on barrier outside (Fig. 6). This condition is satisfied when the width of the proton or deuteron level in the exchange potential well is greater than the distance between the resonant $R$-levels on the inner side of the barrier. This is realized when the exchange potential well is wide and not deep. A thermal proton or deuteron, as well as a thermal neutron, is captured by the nucleus into this potential well at a distance $L$. When a neutron or proton, deuteron is captured, they transfer between levels in a potential pit or in a potential well of a nuclear molecule with electromagnetic radiation in the range from thermal waves to $X$-ray quanta. Protons, deuterons will radiate in the same range when braking in the Coulomb field of the nucleus (Fig. 6) [37-40, 43]. 
Thus, the resonant interference exchange interaction between a proton or deuteron and the $R$-state creates an exchange potential well on the Coulomb barrier of nucleus $A$. As a result, a nuclear molecule is formed: $A+\mathrm{p}$ or $A+\mathrm{d}$. The lifetime of such a molecule is determined by the transparency of Coulomb barrier (7). It is obvious that electronic levels in the nuclear molecule ${ }_{Z} A+\mathrm{p}$ shift relative to electronic levels in the atom ${ }_{\mathrm{Z}+1} B$, where $Z$ is the nuclear charge.

At a high concentration of protons (deuterons) per one nucleus of the main element a second proton or a second deuteron can get into in exchange well: $A+\mathrm{p}+\mathrm{p}$ or $A+\mathrm{d}+$ d. Protons-fermions in the ground state will have antiparallel spins in the well, deuteronsbosons can have differently directed spins, since deuteron levels are closely spaced triplet states $\left(S_{\mathrm{d}}=1, m_{\mathrm{S}}=+1,0,-1\right)$.

As a result, a possibility for cold fusion reactions opens up (Fig. 6):

$$
\begin{aligned}
& \mathrm{d}+\mathrm{d}+A \rightarrow{ }^{4} \mathrm{He}+A+23.8 \mathrm{MeV} \\
& \mathrm{p}+\mathrm{p}+A \rightarrow \mathrm{d}+\mathrm{e}^{+}+\nu_{\mathrm{e}}+A+0.42 \mathrm{MeV} .
\end{aligned}
$$

The reaction (16) with positron emission can be strongly decelerated because of the need to fulfill the angular momentum conservation law. Strong deceleration of reaction (16) opens up the possibility of a reaction with orbital $K$-electron capture:

$$
\mathrm{p}+\mathrm{p}+\mathrm{e}^{-}+A \rightarrow \mathrm{d}+\nu_{\mathrm{e}}+A+1.44 \mathrm{MeV} \text { (17). }
$$

It is clear that, other things being equal, the registration of released energy and the detection of deuterons during that reaction are difficult compared with reactions (15 and 16) $\left(\mathrm{e}^{+}+\mathrm{e}^{-} \rightarrow\right.$ $2 \gamma+1.022 \mathrm{MeV})$. Perhaps, it is for that reason that the reaction (17) cannot be registered in experiments.

Fig. 7 shows that for a proton or deuteron located in the exchange well, the transparency of the Coulomb barrier increased (7) as the height and width of the barrier decreased. In addition, the permeability of the barrier should additionally increase due to the existing resonant levels on the inner side of the Coulomb barrier [41]. Recall that the probability of absorption of a neutron by a nucleus, among other things, depends on the probability of gamma transitions $(\sim 8 \mathrm{MeV})$, when the neutron "descends" to the ground state from upper level at which it was captured. The probabilities of gamma transitions are strongly suppressed, since they are carried out by electromagnetic, rather than strong, interactions. In the case when there are two protons (deuterons) in the exchange well, the probability of tunneling one of the protons through the Coulomb barrier directly into the ground state of the compound nucleus without gamma transitions appears (Fig. 6). This reaction becomes possible due to the strong interaction of protons with the nucleus and the reaction pulse transfer to the second proton:

$$
\mathrm{p}+\mathrm{p}+{ }_{\mathrm{z}} A \rightarrow{ }_{\mathrm{z}+1} B+\mathrm{p}+Q
$$

where $Q$ is energy released in the reaction. With such a transition to ground state, the ${ }_{\mathrm{Z}+1} B$ nucleus may turn out to be beta $\beta^{+}$-radioactive nucleus. Since the disturbing potential of the exchange interaction includes a weak interaction $W$, the reaction can proceed with the capture of the orbital $K$-electron. Then the reaction produces a stable isotope.

$$
\mathrm{p}+\mathrm{p}+\mathrm{e}^{-}+{ }_{\mathrm{Z}} A \rightarrow{ }_{\mathrm{Z}} \text { Astable }+\mathrm{p}+Q
$$

It is such reactions $(18,19)$ that can explain the appearance of stable copper isotopes in the $\mathrm{Ni}+\mathrm{p}$ reaction with a changed isotope ratio in nickel [19, 20].

Obviously, with the preservation of coefficients $U K^{4} M^{2}$, the barrier transparency also increases at nuclear charge decrease. Fig. 8 shows the formation of an exchange potential well for titanium Ti, and Fig. 9 - for lithium Li with coefficients $U K^{4} M^{2}=-2 \mathrm{MeV}$ and $U K^{4} M^{2}$ $=-0.7 \mathrm{MeV}$, respectively. The red line in Fig. 8,9 is nuclear potential plus Coulomb potential of $\mathrm{Ti}+\mathrm{p}$ and $\mathrm{Li}+\mathrm{p}$, the blue line is the RIEX interaction potential (values of $400 \mathrm{fm}$ and 300 fm and $U K^{4} M^{2}$ are chosen arbitrarily) and the green line is summarized potential.

For lithium, the resonant proton is surprisingly absorbed by the nucleus without 


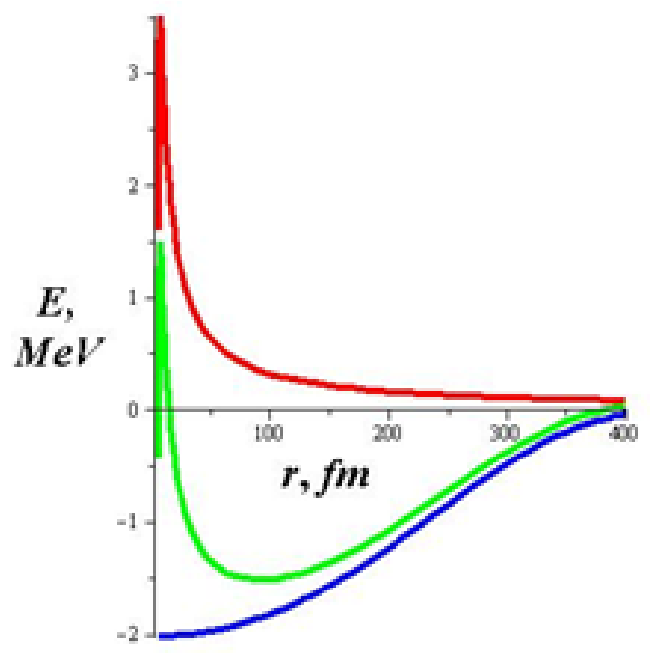

Fig. 8. Red line - nuclear plus Coulomb potentials Ti+p; blue - RIEX potential Ti-p; green - total potential Ti+p. Coulomb barrier, like a resonant neutron, if the coefficient $\left|U K^{4} M^{2}\right|$ exceeds the height of Coulomb barrier $\left|U K^{4} M^{2}\right|>0.5 \mathrm{MeV}$ (Fig. 9). For lithium and boron $\left({ }_{5} B\right)$, exchange barrier or barrier-free transitions are all the more likely because they can occur with one proton or one deuteron, since the compound nucleus decays into fragments [20, 42]. No energy mediator " $Y$ " is needed in these reactions. The height of boron Coulomb barrier is $0.9 \mathrm{MeV}$. Thermal neutron capture cross-section is $\sigma_{\mathrm{T}}\left({ }^{6} \mathrm{Li}\right)=940$ barn, $\sigma_{\mathrm{T}}\left({ }^{10} B\right)=3840$ barn (see Table).

${ }^{6} \mathrm{Li}+\mathrm{d} \rightarrow{ }^{4} \mathrm{He}+{ }^{4} \mathrm{He}+22.37 \mathrm{MeV}$

${ }^{7} \mathrm{Li}+\mathrm{p} \rightarrow 2 \cdot{ }^{4} \mathrm{He}+17.35 \mathrm{MeV}$,

${ }^{10} \mathrm{~B}+\mathrm{d} \rightarrow 3 \cdot{ }^{4} \mathrm{He}+17.9 \mathrm{MeV}$,

${ }^{11} \mathrm{~B}+\mathrm{p} \rightarrow 3 \cdot{ }^{4} \mathrm{He}+8.68 \mathrm{MeV}$.

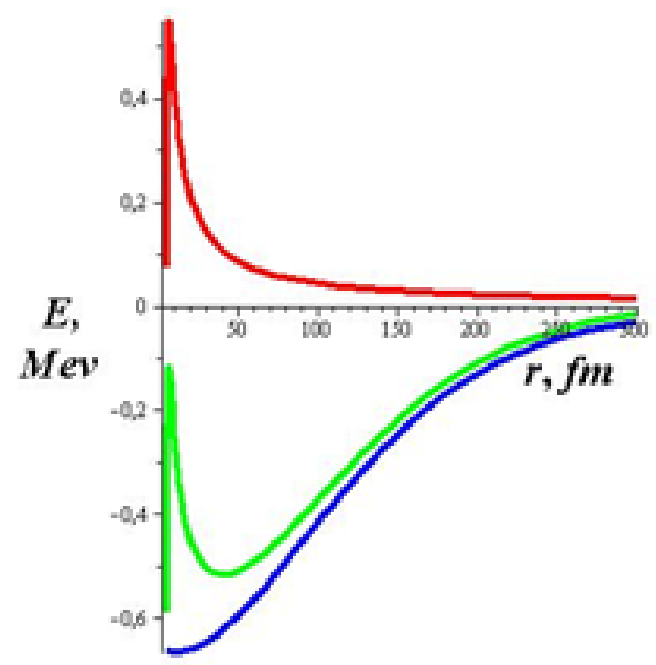

Fig. 9. Red line - nuclear plus Coulomb potentials Li+p; blue - RIEX potential Li-p; green - total potential Li+p.
However, both for neutrons and for protons and deuterons, there seems to be a limited number of isotopes that have significant potential for RIEX interaction with a large $U K^{4} M^{2}$ coefficient and which are capable of forming a deep exchange nuclear well with protons and deuterons. Most isotopes have probably small coefficient $K: K<1$ and $K<<1$. Therefore, not a deep potential well, but a shallow potential "pool" is formed in some of them as a result of the RIEX interaction (Fig. 10). The bottom of such a "pool" and corresponding levels of protons (deuterons) in it can exceed the zero line of potential energy. The lifetime of such a nuclear molecule, the residence time of protons and deuterons in such a "pool" are supposed to be limited. And if external conditions change and do not allow to reproduce again unstable nuclear molecules, they disappear in some time [43].

Since the proton and neutron in the deuteron have a noticeable probability of $\sim 65 \%$ to be outside the nuclear potential well, i.e. to stay in a quasi-free state for some time, the proton and neutron can be assumed to interact independently with their $R$-states. It follows from this that cold fusion reactions on deuterons should have noticeable cross-sections on isotopes, for which the neutron resonance absorption cross-sections are also significant (see Table).

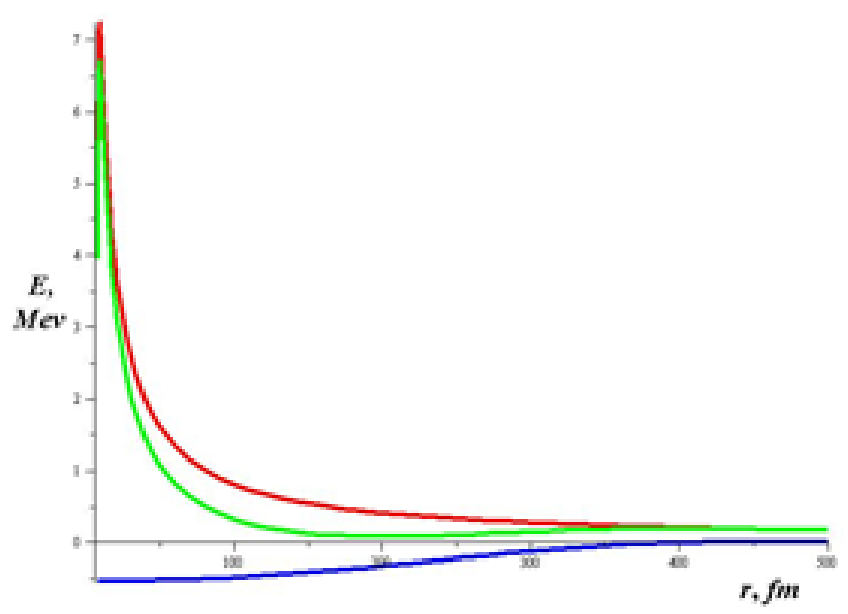

Fig. 10. Formation of a potential "pool". Red line - nuclear plus Coulomb potentials ${ }_{56} \mathrm{Ba}$; blue - RIEX potential; green - total potential. 
The possibility of a proton and a neutron to be outside the nuclear potential well of the deuteron can be interpreted as the existence of RIEX interaction between them. Neutron and proton spins in the deuteron are parallel, and their spatial wave function is symmetric. At that, their total wave function is antisymmetric because of the antisymmetric isotopic spin part of their wave function - $n, p$.

Based on the isotopic invariance of strong interaction, it can be assumed that proton can resonantly interact with neutron $R$-levels, both located behind the Coulomb barrier (Fig. 6) and those of isotopes with significant resonant absorption of neutrons (see Table) [43].

\section{CONCLUSION}

Several conditions should be met for the implementation of cold fusion reactions. First of all, it is necessary that the molecules of hydrogen or deuterium dissociate into atoms in a condensed solid or liquid matter.

It also requires that hydrogen or deuterium atoms in a condensed matter transform into protons or deuterons. It is important that protons or deuterons have maximum possible concentration $(>2)$ per nucleus of main element. The latter is achieved either by electrolysis or electrical discharge by means of saturating the cathode material with protons or deuterons, or by high hydrogen (deuterium) pressure or by nanoclustering the substance, or by its melt, or by aqueous solution of a substance in which hydrogen atoms are present in the form of proton, including biological systems $[12,13,17$, 19, 20, 23-26, 37-40, 42-47].

For CF reactions, it is absolutely necessary that the protons or deuterons incident on the nucleus have R-levels resonant with a compound nucleus. Due to the existence of R-levels between the nucleus and the proton (deuteron), a resonant interference exchange interaction arises, the disturbing potential of which are all types of interactions simultaneously. The disturbing potential that makes the main contribution to the exchange energy is strong interaction. The spatial domain of the resonant interference exchange interaction is related to the wavelength of the proton (deuteron) as $L=$ $K^{4} \lambda_{\mathrm{p}}$. The value of coefficient $K$ depends on the affinity of $\mathrm{R}$-state with a proton (deuteron).

The exchange energy between the proton (deuteron) and the compound nucleus with the $R$-state makes an additional contribution to the energy of their Coulomb and nuclear interaction. Due to this exchange energy, an exchange potential well appears on the Coulomb repulsion barrier. A proton (deuteron), trapped in exchange well, forms a nuclear molecule with a nucleus, and it can tunnel through a reduced Coulomb barrier in the case when a compound nucleus decays into two or three fragments.

The probability of passing through the Coulomb barrier for the proton also increases when a second proton enters the exchange well, which can perform the function of an energy mediator in the reaction.

In the case when there are two protons or two deuterons in the exchange well, they can merge. Thus, cold fusion reactions begin in condensed matter.

The exchange interaction represents a purely quantum effect, which disappears with the limiting transition to classical mechanics. Up to now, the exchange interaction has been considered as an interaction between identical particles. This article argues that the resonant interference exchange interaction does exist. The RIEX interaction is an exchange interaction between any object and resonant $R$-state belonging to any system. RIEX interaction is a development of resonant synchronization principle and its mechanism [48, 49]. All fundamental interactions form simultaneously the disturbing potential of the RIEX interaction: strong, electromagnetic, weak, and gravitational (8). The spatial domain of the resonant interference exchange interaction for all fundamental potentials is determined by the lengths of the wave functions for any resonantly interacting "identical” objects. Due to the RIEX 
interaction, the short-range strong and local weak interactions become "long-range" interactions.

The formulated RIEX interaction goes beyond pure quantum effects. Resonance is nothing else but RIEX interaction.

The world around us consists of identical particles: protons, neutrons and electrons, which in turn form identical objects and identical systems. All types of fundamental interactions occur between identical particles, objects, systems including the RIEX interaction. The RIEX-interaction on a par with other interactions creates the Universe. It is a Universal interaction because it includes all other Four interactions.

Resonant Interference Exchange interaction is the Fifth type of interaction.

\section{REFERENCES}

1. Proceedings of the 1-25th Russian Conferences on Cold Transmutation of Nuclei of Chemical Elements. Dagomys, Sochi, 1993-2018.

2. Proceedings of the 1-21th International Conferences on Condensed Matter Nuclear Science (Cold Fusion) - ICCF. USA, Canada, France, Italy, Japan, China, Korea, India etc. 1990-2018.

3. Balakirev VF, Krymsky VV, Bolotov BV, Vasilieva NV, Vachaev AV, Ivanov NI, Kazbanov VI, Pavlova GA, Solin MI, Trofimov VI, Urutskoev LI. V zaimoprevrashcheniya khimicheskikh elementov [Mutual transformation of chemical elements]. Ekaterinburg, Urals Branch of RAS Publ., 2003, 64 p.

4. Radioelectronics. Nanosystems. Information Technologies (RENSIT), 2017, 9(1); DOI: 10.17725/rensit.2017.9.

5. Zhurnal formiruyushchikhsya napravleniy nauki [nternational Journal of Unconventional Science (IJUS)], 2017, No.17-18 (in Russ.); http://www.unconv-science.org/.

6. Prosvirnov AA, editor of the LENR-site; http://www.lenr.seplm.ru/.

7. Mishinsky GV, Kuznetsov VD, Penkov FM. Nizkoenergeticheskaya transmutatsiya atomnykh yader khimicheskikh elementov. Raspredelenie po elementam $\mathrm{v}$ produktakh transmutatsii. nukleosintez [Low energy transmutation of atomic nuclei of chemical elements. Element distribution in the products of low energy transmutation. Nucleosynthesis]. IJUS, 2017, No.17-18 (5):61-81 (in Russ.).

8. Mishinsky GV. Spin electron condensate. Spin nuclide electron condensate. RENSIT, 2018, 10(3):411-424. DOI: 10.17725/ rensit.2018.10.411.

9. Mishinsky GV. Non-Coulomb nuclear reactions of transatoms. Stellar energy and nucleosynthesis. RENSIT, 2018, 10(1):35-52. DOI: $10.17725 /$ rensit.2018.10.035.

10. Mishinsky GV. Atom in a strong magnetic field. Transformation of atoms to transatoms. RENSIT, 2017, 9(2):147-160. DOI: 10.17725/ rensit.2017.09.147.

11. Mishinsky GV. Multinuclear reactions in condensed helium. RENSIT, 2017, 9(1):94105. DOI: 10.17725/rensit.2017.09.094.

12. Fleishmann M, Pons S, Hawkins M. Electrochemically induced nuclear fusion of deuterium. J. Electroanal. Chem. 1989, 261:301-308.

13. Filimonenko IS. Demonstratsionnaya termoemissionnaya ustanovka dlya yadernogo sinteza [Demonstrational therm-emission device for nuclear fusion]. Materials 3 Sci. Symp. "Perestroyka estestvoznaniya [Restructuring of Natural history]"-92, Volgodonsk, Russia, 1719 apr. 1992 (in Russ.).

14. Iyengar PK. Cold Fusion Results in BARC Experiments. Proc. 5 Intern. Conference On Emerging Nuclear Energy Systems (ICENES), Karlsruhe, FRG, Singapore, 1989; Iyengar PK. and Srinivasan M. Overview of BARC Studies in Cold Fusion. Proc. 5 Intern. Conference on Cold Fusion (ICCF-5), 1990, Utah, Salt Lake City.

15. McKubre MCH. CMNS Research - Past, Present and Future. J. Condensed Matter Nuclear Science, 2017, 24:15-24.

16. Miles MH. Heat and helium production in cold fusion experiments. 2nd Int. Conf. on Cold Fusion "The Science of Cold Fusion", Como, Italy: Societa Italiana di Fisica, Bologna, Italy, 1990. Miles MH, Hollins RA, Bush BF, Lagowski JJ, Miles RE. J.Electroanal.Chem., 1993, 346:99. Miles MH, Proc. ICCF-10, 2003, Cambridge. 
17. Karabut AB, Kucherov YaR, Savvatimova IB. Nuclear product ratio for glow discharge in deuterium. Phys.Letters A, 1992, 170: 265-272. Savvatimova IB, Karabut AB. The products of nuclear reactions recorded at the cathode after experiments in a glow discharge in deuterium. Povernoct ' [Surface Investigation: X-Ray, Synchrotron and Neutron Techniques], 1996, 1:63-75-81.

18. Focardi S, Habel R and Piantelli F. Anomalous Heat Production in Ni-H Systems. Nuovo Cimento, 1994, 107(1):163-166.

19. Parkhomov AG, Alabin KA, Andreev SN, Zabavin SN, Sobolev AG,Timerbulatov TR. Nickel-hydrogen reactor: heat generation, isotopic and elemental composition of fuel. RENSIT, 2017, 9(1):74-93. DOI: 10.17725/ rensit.2017.09.074.

20. Levi G, Foschi E, Höistad B. Observation of abundant heat production from a reactor device and of isotopic changes in the fuel. Http:// www.sifferkoll.se/sifferkoll/wp-content/ uploads/2014/10/LuganoReportSubmit.pdf.

21. Kalman P, Keszthelyi T. Lattice effect in solid state internal conversion. Phys. Rev. C, 2009, 79(0316026):1-4.

22. Bazhutov YuN. Possible generation of neutrons birsts in framework of erzion model and their possible registration; Essence of erzion model. Proc.13th Intern. Conference on Condensed Matter Nuclear Science. Moscow, MITI, 2008:581-589.

23. Arata Y, Zhang Y-C. Characteristics of Compact and Practical "Solid Deuterium Nuclear Fusion Reactor". Proc. 10th Intern. Conference on Cold Fusion, 2003, Cambridge, Massachusetts, USA.

24. Arata Y and Zhang YC. Formation of Condensed Metallic Deuterium Lattice and Nuclear Fusion. Proc. Jpn. Acad., Ser. B, 2002, 78:57.

25. Nesvizhevsky VV, Voronin AYu, Lambrecht A, Reynaud S, Lychagin EV, Muzychka AYu, Strelkov AV. Observation of quantum levitation of nanoparticles by the method of ultracold neutrons. Crystallography, 2013, 58(5):730-736 (in Russ.).
26. Didyk AYu, Wisniewski R. Nuclear reactions, induced by gamma quanta, in palladium saturated with deuterium surrounded by dense deuterium gas. Euro. Phys. Lett., 2012, 99, 22001:1-6.

27. Rukhadze AA, Grachev VI. LENR in Russia. RENSIT, 2017，9(1):5-7. DOI: 10.17725/ rensit.2017.09.005.

28. Wendt GL, Irion CE. Experimental attempts to decompose tungsten at high temperatures. J. of the American Chemical Society, 1922, 44(9):1887-1894.

29. Smits A, Karssen A. Vorläufige Mitteilung über einen Zerfall des Bleiatoms. Naturwissenschaft, 1925, 13:699. Ein Zerfall des Bleiatoms, Z. Elektrochemie, 1926, 32:577-586.

30. Mills R, Ray P. Extreme ultraviolet spectroscopy of helium-hydrogen plasma. J. Phys. D:Appl. Phys., 2003, 36:1535-1542.

31. Tsyganov EN. Kholodny yaderny sintez [Cold fusion]. Yadernaya firika, 2012, 75(2):174-180 (in Russ.).

32. Vlasov NA. Neytrony [Neutrons]. Moscow, Nauka Publ., 1971, 552 p.

33. Heisenberg W. Über die Spektra von Atomsystemen mit zwei Elektronen. Z. Phys., 1926, 39(7):499-518.

34. Matveev AN. Atomnaya firika [Atomic physics]. Moscow, Vysshaya shkola Publ., 1989, 439 p.

35. Gurevich VL, Pogorelsky MJ, Chaly VP. Dvukhyadernoe atom-svyazannoe sostoyanie protona i tyazhelogo atoma [Binuclear atombound state of proton and heavy atom]. Zhurnal tekhnicheskoy firiki, 2009, 79(2):1-5 (in Russ.).

36. Polikanov SM. Izomeriya formy atomnykh yader [Isomerism of atomic nuclei form]. Moscow, Atomizdat Publ., 1977, 200 p.

37. Vysotskii VI, Kornilova AA, Vasilenko AO, Krit TB, Vysotskyy MV. The prediction, observation and study of long-distant undamped thermal waves generated in pulse radiative processe. Nuclear Instruments and Methods in Physics Research B, 2017, 402:251-255.

38. Vysotskii VI, Kornilova AA, Sysoev NN. $\mathrm{X}$-ray radiation by cavitation of rapid liquid jet. RENSIT, 2010, 2(1-2):57-69. 
39. Klimov A, Grigorenko A, Efimov A, Evstigneev N, Ryabkov O, Sidorenko M, Soloviev A, Tolkunov B. High-energetic nanocluster plasmoid and its soft X-radiation. Proc.19th Intern. Conference on Condensed Matter Nuclear Science, 2015. J. Condensed Matter Nucl. Sci., 2016, 19:145-154.

40. Klimov A. Energy Release and Transmutation of Chemical Elements in Cold Heterogeneous Plasma. Proc.19th Intern. Conference on Condensed Matter Nuclear Science. Padua, Italy, 2015. J. Condensed Matter Nucl. Sci., 2016, 19:155-163.

41. Demikhovsky VYa, Vugalter GA. Fizika kvantovykh nizkorazmernykh struktyr [Physics of quantum low-dimensional structures]. Moscow, Logos Publ, 2000, 248 p.

42. Vysotskii VI, Kornilova AA, Chernysh VS, Gavrilova ND, Lotonov AM. Stimulated (B11p) LENR and Emission of Nuclear Particles in Hydroborates in the Region of Phase Transfer Point. J. Condensed Matter Nucl. Sci., 2014, 13:608-614.

43. Klimov AI. Will Russia be among the leaders of new energy technologies? IA REGNUM, 2019, (in Russ.); https://regnum.ru/news/2625783. html.

44. Didyk AYu, Wiśniewski R, WilczyńskaKitowska T, Mishinsky GV, Semin VA. Synthesis of chemical elements under irradiation by bremsstrahlung gamma-rays of palladium in condensed gases. RENSIT, 2019, 11(2):143-160.

45. Andreev SN, Shafeev GA. Nelineynoe tushenie radioaktivnosti vodnykh rastvorov soley nuklidov pri lazernoy ablyatsii nanochastits metallov [Onlinear quenching of the radioactivity of aqueous solutions of salts of nuclides by laser ablation of nanoparticles of metals]. RENSIT, 2017, 9(1):65-73. DOI: 10.17725/rensit.2017.09.065.

46. Astakhov BA. Nekotoryye predlozheniya o puskovom mekhanizme CF. [Some suggestions about the trigger mechanism CF]; http:// lenr2019.ru/.

47. Kornilova AA. Vysotskii VI. Synthesis and transmutation of stable and radioactive isotopes in biological systems. RENSIT, 2017, 9(1):52-64. DOI: 10.17725/rensit.2017.09.052.

48. Gareev FA, Gareeva GF, Zhidkova IE. Mekhanizm protsessa kholodnoy transmutatsii yader khimicheskikh elementov [The mechanism of the process of cold nuclear transmutation of chemical elements]. Geoinformatics, 2003, 1:51-53. (in Russ.).

49. Gareev FA, Gareeva GF, Zhidkova IE. Printsip rezonansnoy sinkhronizatsii dlya proryvnykh tekhnologiy [The principle of resonant synchronization for breakthrough technologies]. Collected Works of the Sustainable Innovative Development Department of the International University of Nature, Society and Man "Dubna". K. 89-1, Moscow, RAEN Publ., 2007:138-154. 